\title{
Development of faster front end electronics for the SciCRT detector at Sierra Negra, Mexico
}

\author{
Marcos Anzorena ${ }^{* a}$, J.F.Valdés-Galicia ${ }^{a}$, Rocío García ${ }^{a}$, Yutaka Matsubara ${ }^{b}$, \\ Yoshinori Sasai $^{b}$, Tetsuya Kawabata ${ }^{b}$, Ernesto Ortiz $^{c}$, L.X.González ${ }^{d}$, Octavio \\ Musalem $^{a}$, Alejandro Hurtado $^{a}$, Marco Barrantes $^{a}$, Roberto Taylor $^{a}$, Yoshitaka Itow $^{b}$, \\ Takashi Sako $^{b}$, Akira Tsuchiya ${ }^{b}$, Kazuoki Munakata $^{e}$, Chihiro Kato $^{e}$, Yoshiaki \\ Nakamura $^{e}$, Takahiro Oshima ${ }^{e}$, Toshiki Koike ${ }^{e}$, Shoichi Shibata $^{f}$, Akitoshi Oshima $^{f}$, \\ Hisanori Takamaru $^{f}$, Hiroshi Kojima ${ }^{g}$, Harufumi Tsuchiya ${ }^{h}$, Kyoko Watanabe $^{i}$, \\ Masayoshi Kozai ${ }^{j}$, Tatsumi Koi ${ }^{k}$ \\ E-mail: anzorenalgeofisica.unam.mx
}

The SciBar Cosmic ray telescope (SciCRT) is installed on the top of the Sierra Negra volcano with the main goal of observing solar neutrons to investigate the ion acceleration process during solar flares. Using scintillator bars as a medium to stop energetic particles, the SciCRT is capable of recording both energy deposited on the bars and direction of the incoming particles with high resolution. The original DAQ system was used in neutrino oscillation experiment (low event rate), therefore operation of the electronics on cosmic ray experiment is limited. To improve the SciCRT performance as a solar neutron telescope, development of custom made DAQ electronics is essential. Our first step onto this task was the design and construction of a new fast readout back-end board using SiTCP. The installation of this new system on Sierra Negra and its further improvement on the data acquisition for the detector will be analyzed on separate paper on this conference. The development of new front end electronics is the next stage of the upgrading process. To achieve this goal, we are developing new electronics applying the time over threshold (ToT) technique, using a FPGA to process the signal from one 64-channel multi anode photomutiplier tube (MAPMT). In this paper we will present the details of this new system and several tests performed to guarantee its proper operation to detect solar neutrons.

35th International Cosmic Ray Conference

10-20 July, 2017

Bexco, Busan, Korea

\footnotetext{
* Speaker.

${ }^{\dagger a}$ Instituto de Geofísica, Universidad Nacional Autónoma de México, Ciudad de México, 04510, México.

${ }^{\dagger b}$ Institute for Space-Earth Environmental Research, Nagoya University, Furo-cho, Chikusa-ku, Nagoya 464-8601,

${ }^{\dagger c}$ Instituto de Ciencias Físicas, Universidad Nacional Autónoma de México, Cuernavaca, Morelos, 62210, México.

${ }^{\dagger}{ }^{d}$ SCiESMEX, Instituto de Geofísica, Unidad Michoacán, Universidad Nacional Autónoma de México, Michoacán Morelia, 58190, México.

${ }^{\dagger}$ Department of Physics, Shinshu University, Asahi, Matsumoto, 390-8621, Japan.
} Japan. 


\section{Introduction}

The Sun is capable of accelerating particles at relativistic energies during solar flares. This makes the Sun an excellent laboratory to study particle acceleration mechanisms. Exactly how the acceleration process occurs is still an open problem. Observing charged solar particles accelerated during intense solar flares offers one possibility to study the problem, however, these are delayed and modulated on its travel to Earth by the solar and interplanetary magnetic fields. Observation of secondary neutral particles avoids such complexity. This was the motivation behind the installation of solar neutron telescopes (SNT) around the world. The telescope are installed at high altitude in order to minimize the attenuation of neutrons by the atmosphere. SNTs had been successful observing solar neutron events, however the development of new type detector with larger effective area to improve the statistic significance and better energy resolution it is need to fully extract the information from solar neutrons. The new detector is the SciCRT.

The SciCRT will be also integrated as part of the Global muon detector network, with the goal of monitoring nucleonic components on galactic cosmic rays (GCRs) to study their directional anisotropy observed at the Earth. The continuous observation of GCRs provides valuable information about interplanetary shocks and coronal mass ejections.

\section{The SciBar cosmic ray telescope}

The SciBar detector is a particle tracker able to record deposited energy along the path. SciBar is composed of 14848 scintillator bars, of dimensions $1.3 \times 2.5 \times 300.0 \mathrm{~cm}^{3}$, arranged on horizontal $X-Y$ planes orthogonal to each other. Each plane is made by 116 strips, piled together to form a total of 64 layers. Photons generated by the energy deposition of incident radiation on the telescope travel through the Wavelength shifting fiber (WLS-fiber) installed on every strip. One end of the WLS-fibers is painted white to increase photon collection efficiency. The other end is coupled to a multi-anode photomultiplier tube (MAPMT), which process the incoming optical signal from an array of 64 bars.

SciCRT is capable of detecting muons using four-fold coincidence from top and bottom layers as trigger. Neutral particles are identified when a track is registered in the middle of the detector without triggering any muon layer. The energy deposited is measure by the charge generated at the output of the MAPMTs (ADC data). Scaler data for neutral particles is also obtained, recording the number of hit signals on each MAPMT in anti-coincidence with the muon layers.

The DAQ system of the detector uses a combination of application specific integrated circuit (ASIC) and VME modules. Furthermore, the system may be separated into three sub-systems: front end board (FEB), back-end board (BEB) and trigger board (TRGB). FEBs are reponsable for signal

\footnotetext{
${ }^{f}$ College of Engineering, Chubu University, Kasugai 487-8501, Japan.

${ }^{g}$ Faculty of Engineering, Aichi Institute of Technology, Toyota 470-0392, Japan.

${ }^{h}$ Japan Atomic Energy Agency, 2-4 Shirakata Shirane, Tokai-mura, Naka-gun, Ibaraki 319-1195, Japan.

${ }^{i}$ National Defense Academy of Japan, 1-10-20 Hashirimizu, Yokosuka, Kanagawa 239-8686, Japan.

${ }^{j}$ Institute of Space and Astronautical Science, Japan Aerospace Exploration Agency, Sagamihara, Kanagawa 2525210, Japan.

${ }^{k}$ SLAC National Accelerator Laboratory, Menlo Park, CA 94025-7015, USA.
} 


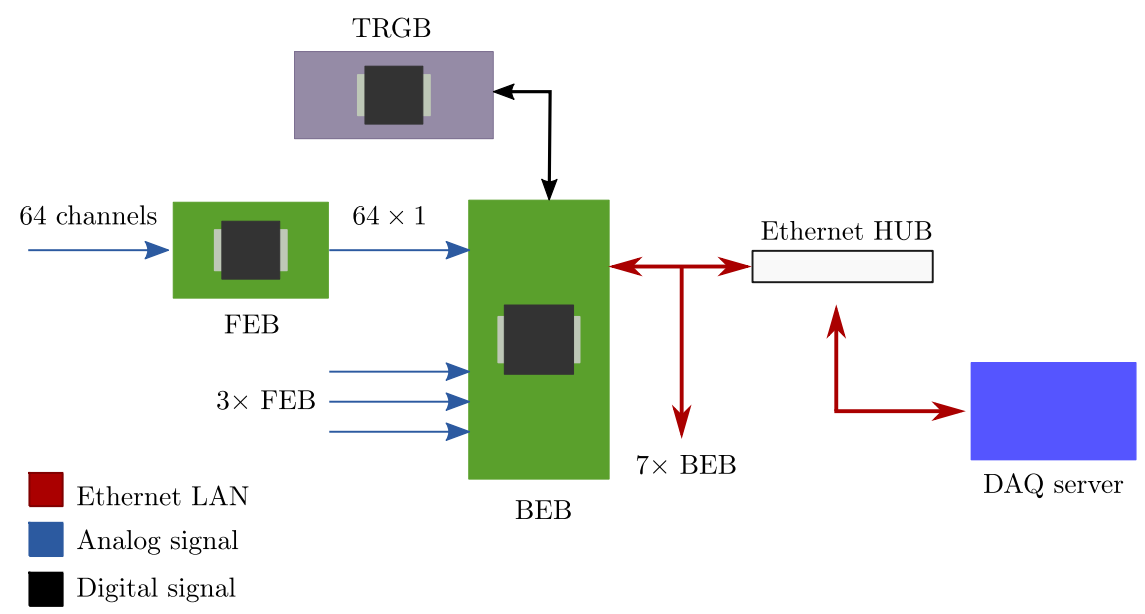

Figure 1: Schematic view of the new robust DAQ system installed on July 2015.

conditioning of MAPMTs. Each FEB is capable of processing 64 channels from MAPMT. BEBs perfom analog digital convertion (ADC) on the signal from FEBs and transfer this information to DAQ servers. The TRGB is used to trigger other modules to capture cosmic rays events. A more detailed explanation of these is found on [] [1].

Because the electronic system was originally designed for an accelerator experiment, the specific requirements depart from our goals as a Solar neutron/cosmic ray experiment. In summary the requirements of the electronic DAQ system for SciCRT to achieve its full performance are [1]:

- Increase the maximum transfer rate for ADC data read from MAPMTs.

- Eliminate the effect of long dead time on scaler data.

- Optimize the system to operate on severe environment conditions (4600 $\mathrm{m}$ above sea level).

Motivated by this, we designed and installed an improved version of the DAQ system on July 2015 on the muon layers and 1/8 of the detector. The new DAQ system uses SiTCP [2] (a hardware network processor implemented on FPGA) based BEBs, to transfer the charge information from 4 FEBs to one DAQ server. A schematic diagram of this system is shown on figure 1. An evaluation on the performance of this improved DAQ system is presented on a separate paper [3].

\section{Development of new front end electronics}

The next step in the upgrading process is the development of new front end electronics. Even though the performance of our front end electronics in solar neutrons events has been evaluated throughout Monte Carlo simulation as satisfactory [1,4], current design of the front end boards is based on ASIC, enabling excellent energy resolution and very small board size at the expense of high production costs per board. This fact is aggravated by the large number of boards need in the installation of the full SciCRT. Operating such system at high altitude is not sustainable on a long term, since the severe weather conditions reduce the useful life of each electronic module, making the construction of spare units a priority in our project. 

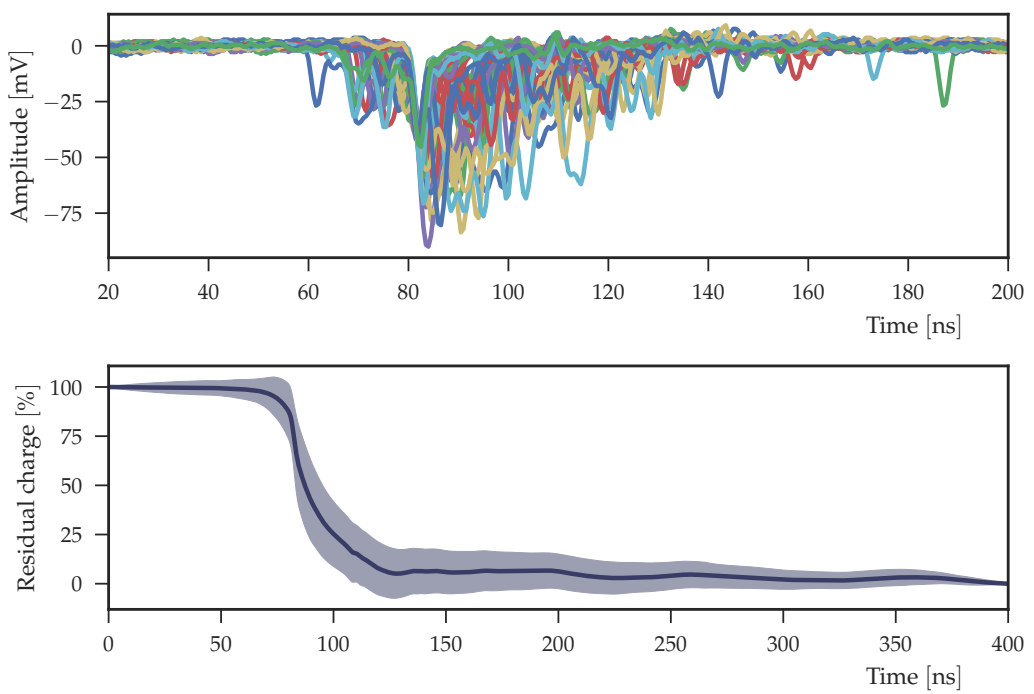

Figure 2: Figure showing the behavior of current signals from one of the MAPMT on the SciCRT. The top panel shows signals from one MAPMT. The bottom panel is shows the residual charge as a function of time. The blue thick line represents the mean value, the light shaded area represents the $\pm \sigma$ interval.

Moreover, due to the limited number of currently existing FEB, the full installation of the SciCRT is not possible. This situation is not acceptable as the effective area of the detector is diminished and overall sensitivity to solar neutrons is decreased. Considering this issues we started the development of a new low cost front end board for fully realizing the capabilities of SciCRT as an improved SNT.

Original FEB integrates the functions of charge sensitive preamplifier, pulse shaping, sample and hold, discriminator and analog multiplexer. The output from the charge sensitive preamplifier is divided in two signal paths. The first path, is for energy estimation and comprises a slow shaper, sample and hold and analog multiplexer. The second path is constituted by a fast shaper, discriminator and logical OR circuit. This path is useful for trigger functions.

Considering that most of the functions of the FEB are performed on analog signals, the only possibility to produce a low cost and effective design is the use of discrete components. The disadvantage of such method is the increase on board size. To countermeasure this effect we decided to use only one signal path with a fast shaper. Since this reduces the resolution on energy, we performed an analysis on the output signals from the MAPMT installed on the SciCRT to calculate the percentage of lost charge when using only fast shaper. Figure 2 presents the results of this analysis. The top panel shows a small example set of 50 waveforms from cosmic rays interacting on one scintillator bar. The waveforms were captured with oscilloscope at the bottom layer of the detector (muon layers). The bottom panel shows the residual charge of the pulses as a function of time. Synchronizing all pulse waveforms to start at $50 \mathrm{~ns}$, it can be seen from the figure that more than 90 of the total charge in the pulses is deposited in a time window of $100 \mathrm{~ns}$. This will be one of the design constrains of the pulse shaping network.

To further the reduce the complexity of the analog processing unit, we have adopted an alternative method to traditional pulse amplitude reading called Time over threshold [5]. In this technique 


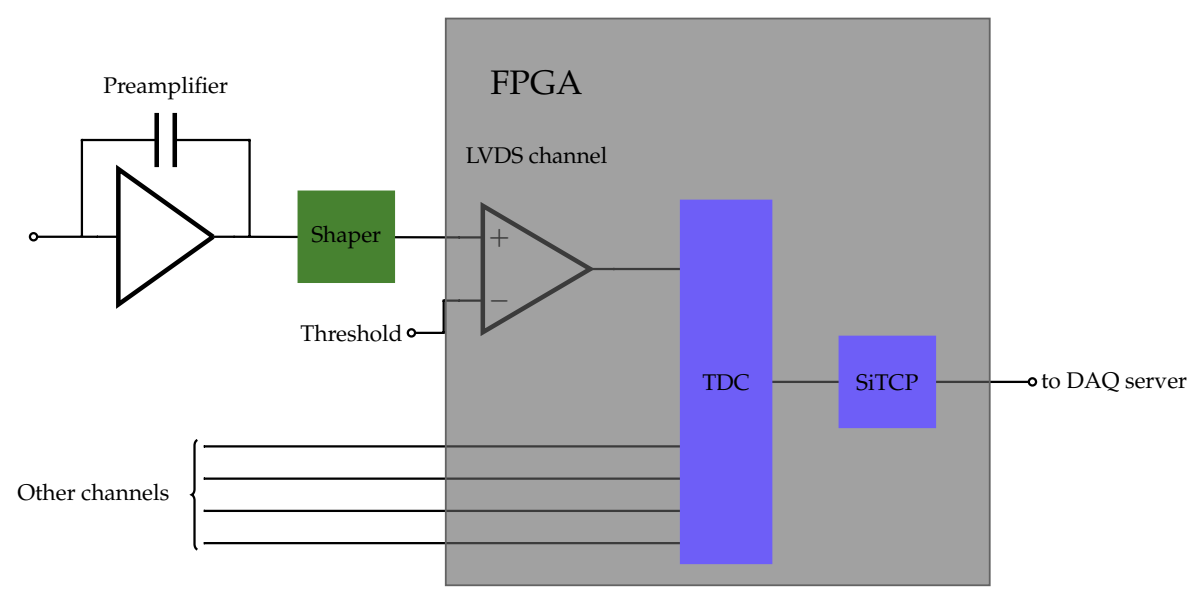

Figure 3: Block diagram of the new front end electronics considering the integration of SiTCP on the board.

the signals coming from the shaping network pass through a level discriminator and generate a time varying digital signal $T_{O T}$. The width of the signal has a logarithmic relationship with the amplitude of pulse and therefore it may be used to measure the deposited charge. To convert the time information to a digital value representing the charge, we must employ a time digital converter (TDC). Because the technique is performed on the digital domain, it reduces the number of elements on the analog processing chain. We use a field programmable gate array (FPGA) to integrate most of the functions required by the time over threshold unit. Figure 3 shows the block diagram of the new board we are designing.

An important observation from the figure is that we implement the level discriminators as LVDS receivers on the FPGA. This not only reduces board size but also power consumption and generation of electromagnetic interference [6]. Also in this diagram we are considering the integration of SiTCP to the architecture of the FEB, allowing the direct transfer of ADC data to the DAQ server. Although this option at first seems promising, it has to be carefully studied to actually determine its cost-benefit on the full installation of SciCRT.

The next step in the development is the design of the TDC. Since the TDC must implemented with the FPGA, we need a technique suitable for such kind of devices. We choose the oversampling or interpolation technique [5] because it can be synthesized on the FPGA with few resources and achieve a high time resolution. In this case, time resolution is a very important parameter because it defines the energy resolution of the FEB.

Figure 4 describes the principle of operation of the TDC with the oversampling technique. The converter is composed of two main elements: a coarse counter and a interpolator. The counter is enabled only when a $T_{O T}$ is to be measure. This gives an integer number of clock cycles $\left(n T_{c l k}\right)$ as the time measurement of the signal. Because the coarse counter is limited in is frequency of operation, to improve the rough resolution we use the interpolator circuit.

A fractional time duration $f T_{c l k}$ can be measured using $M$ number of similar clock reference signals with equal period, but a relative phase difference between them, generated inside the FPGA. The interpolator circuit uses a bank of registers (flip-flops) to determine the leading edge of the temporally closest reference clock to the $T_{O T}$ and codifies this information as a binary value. The total time measurement therefore will be $n T_{c l k}+f T_{c l k}$. An example of this is shown on the right 

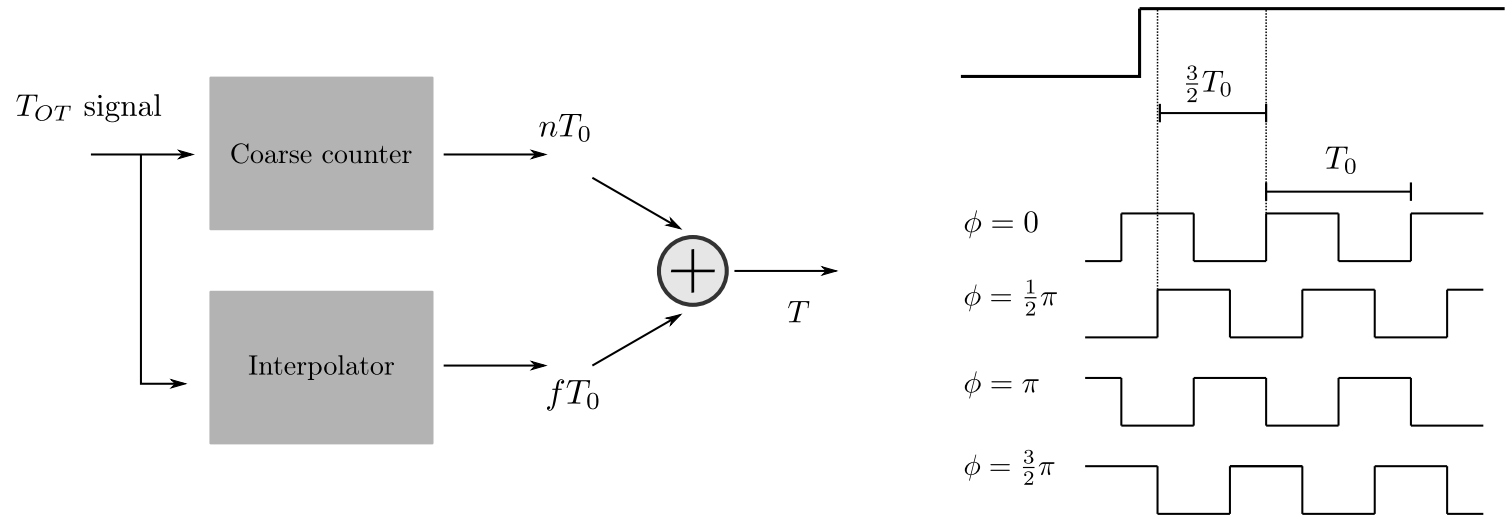

Figure 4: Timing diagram and schematic showing principle of operation of the oversampling technique.

side of figure 4 . Using a total of 4 clock reference signals, with a phase difference of $p i / 4$ between each, the total time resolution of this scheme is $4 \times T_{c l k}$.

\section{Performance of first prototype and future work}

In march 2016 we designed and built one channel of the preamplifier-shaper circuit for the new front end board. A picture of this circuit is shown on figure 5. The preamplifier is designed as a charge sensitive preamplifier with a time constant of $300 \mathrm{~ns}$. The shaping network is composed of a pole-zero cancellation circuit (to avoid undershot) and a fifth order gaussian shaper. The total gain of the circuit is $\approx 90$. Both sections of the circuit are constructed with the operational amplifier ADA4891 from analog devices. This IC was selected because its well balanced between bandwidth product, slew rate and low noise. Moreover, the small packaging of the device allows the construction of a whole preamplifer-shaper circuit with only 2 ICs. The basic performance of the circuit was evaluated with a small prototype of the SciCRT, the miniscibar. The miniscibar is constructed from the same type of scintillator strips and WLS-fiber as the SciCRT but with smaller dimensions. A full description of this detector is found elsewhere [7].

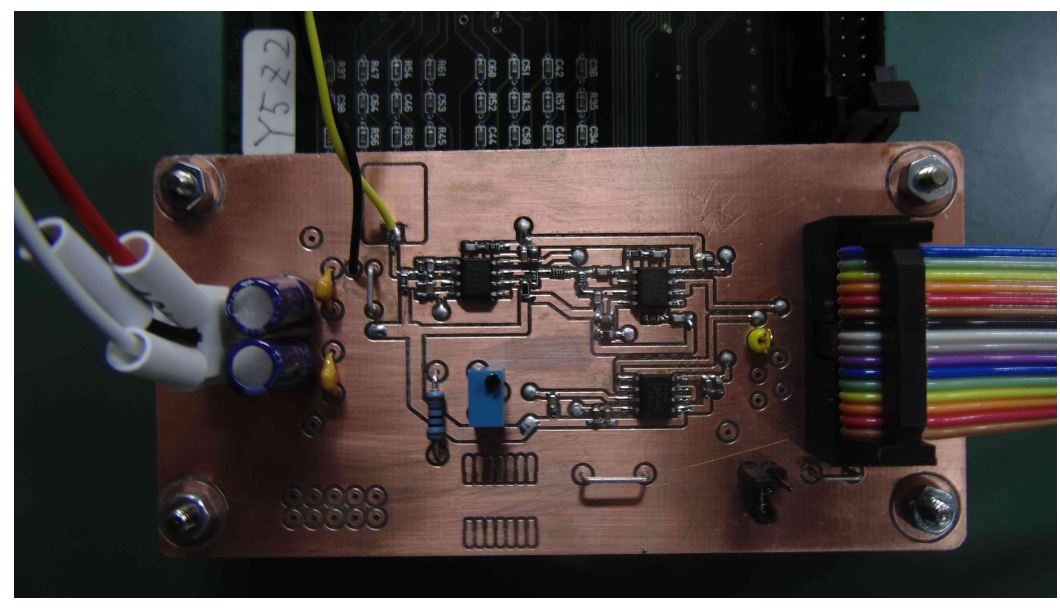

Figure 5: Preamplifier-shaper circuit for the SciCRT. 
With this prototype we manage to detect cosmic ray muons with a counting rate of $15 \mathrm{~Hz}$. This measurement was performed at sea level in the Institute for Space-Earth Environmental research (ISEE) in Nagoya University. The result is consistent with counting rate expected from a detector with the characteristics of miniscibar. On the other hand, one problem we found using this circuit was the baseline shifting of the output from the shaper, caused by the DC coupling of the signal to the FPGA. We will solve this problem on a second version of the circuit.

Because the cosmic ray signal is random in time and energy distribution, to continue the development of the electronics we decided to construct a calibration circuit using a LED. With this circuit we can produce signals with different light intensities and hit rates, allowing us a fine determination of the characteristics of the FEB and the energy resolution. We succeed building a LED driving circuit and installed it, along with a MAPMT, inside the dark room on the Cosmic ray observatory at Mexico city. The LED driver is based on a design presented in [8] and uses a combination of pulse width modulation and forward voltage to control the intensity of the LED. Although we still need to make further tests with this circuit to assert all its parameters, we manage to produce a light intensity similar to a cosmic ray muon interacting on one scintillator strip of the SciCRT [7]. Figure 6 shows this result. This data was obtained using one BEB similar to the installed on the SciCRT.

Currently we are working on the description of the TDC in the FPGA. So far we have been able to test this circuit with some fixed width and frequency signal, but have not yet obtained acceptable results. After we finish the debugging of this system, our goal will be to obtain a similar distribution for the LED on figure 6.

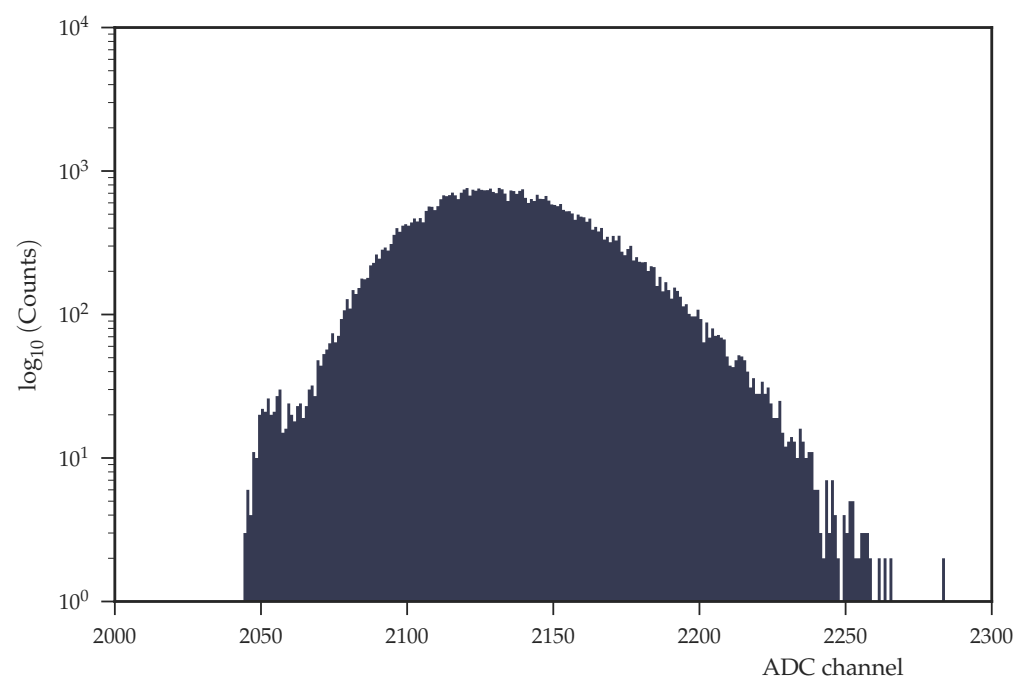

Figure 6: ADC distribution from LED source installed inside a dark box. The operating voltage of the MAPMT is $-800 \mathrm{~V}$. The LED is control with a digital signal of $100 \mathrm{~Hz}$ and $20 \mathrm{~ns}$. The forward current in the LED is $10 \mathrm{~mA}$. 


\section{Summary}

The SciCRT is an improved solar neutron telescope with high sensitivity to solar neutrons and better energy resolution. To improve the performance of the SciCRT we installed a new robust DAQ based on SiTCP. The next step in the upgrading process is the development of FEB electronics. So far we have designed and build a prototype for preamplifier and shaper using discrete components, and manage to detect cosmic ray signal. Our next goal is the development of a time digital converter based on the oversampling technique to achieve high resolution. For the calibration of this device we are using a LED system which is capable of producing signals similar to cosmic ray muons on the SciCRT.

\section{Acknowledgments}

We want to thank the SciBar and SciBooNe experiments teams for allowing us to use the SciBar as a cosmic ray detector. We are also grateful to the National Institute of Astrophysics, Optics and Electronics (INAOE) for allowing us to continue the experiment at the top of Mt. Sierra Negra. Finally we want to thank Mexican Physics Society and local organizing committee of the 35 th ICRC for the funds to attend conference. This work is partially supported by UNAM-PAPIITIN104115 and CONACyT-180727T. Grants from Scientific Research(B) 22340054, Scientific Research(C) 23540348 and Fellows 2434-0054 from JSPS are also acknowledged. This work is also partially supported by the joint research program of the Solar-Terrestrial Environment Laboratory (STEL), Nagoya University, Japan.

\section{References}

[1] Y. Sasai et al., A faster and more reliable data acquisition system for the full performance of the SciCRT, Nuclear Instruments and Methods in Physics Research A 857 (2017) 50-57.

[2] T. Uchida, Hardware-Based TCP Processor for Gigabit Ethernet, IEEE transactions on nuclear science 55 (2008) .

[3] M. Anzorena et al., Current status of SciCRT experiment and its expected future performance, in proceedings of 35th Int. Cosmic Ray Conf. PoS ( ICRC2017) 051, 2017.

[4] Y. Nagai et al., First cosmic-ray measurements by the SciCRT solar neutron experiment in Mexico, Astroparticle physics 59 (2014) 39-46.

[5] W. Yonggang et al., A Linear Time-Over-Threshold Digitizing Scheme and Its 64-channel DAQ Prototype Design on FPGA for a Continuous Crystal PET Detector, IEEE transactions on nuclear science 61:1 (2014) 99-106.

[6] G. Varner, The modern FPGA as discriminator, TDC and ADC, Journal of Instrumentation 1 (2006) P07001.

[7] Nagai, Y., Observation of cosmic rays by the new solar neutron telescope, ph.d. thesis, Nagoya University, 2014.

[8] Hamamatsu Photonics K.K., Opto-semiconductor Handbook. Hamamatsu Photonics K.K., Japan, 2014. 